\title{
Superradiant light scattering and grating formation in cold atomic vapours
}

\author{
N. Piovella ${ }^{\mathrm{a}, *}$, R. Bonifacio ${ }^{\mathrm{a}}$, B.W.J. McNeil ${ }^{\mathrm{b}}$, G.R.M. Robb $^{\mathrm{b}}$ \\ a Dipartimento di Fisica, Università Degli Studi di Milano, INFN and INFM, Sezione di Milano, Via Celoria 16, Milano I-20133, Italy \\ ${ }^{\mathrm{b}}$ Department of Physics and Applied Physics, University of Strathclyde, Glasgow G4 0NG, Scotland, UK
}

Received 3 June 2000; received in revised form 8 September 2000; accepted 7 November 2000

\begin{abstract}
A semi-classical theory of coherent light scattering from an elongated sample of cold atoms exposed to an off-resonant laser beam is presented. The model, which is a direct extension of that of the collective atomic recoil laser, describes the emission of two superradiant pulses along the sample's major axis simultaneous with the formation of a bidimensional atomic grating inside the sample. It provides a simple physical picture of the recent observation of collective light scattering from a Bose-Einstein condensate [Science 285 (1999) 571]. In addition, the model provides an analytical description of the temporal evolution of the scattered light intensity which shows good quantitative agreement with the experimental results of Inouye et al. (C) 2001 Elsevier Science B.V. All rights reserved.
\end{abstract}

PACS: 42.50.Fx; 42.50.Vk; 03.75.Fi

Keywords: Superradiance; BEC; CARL; Atomic grating

\section{Introduction}

Recent experiments by Inouye et al. at MIT [1] have demonstrated the formation of atomic matter waves in a cigar-shaped Bose-Einstein condensate (BEC) pumped by an off-resonant laser beam, together with highly directional scattering of light along the major axis of the condensate. This emission has been interpreted as superradiant Rayleigh scattering, and some theoretical work describing this experiment has been recently published [2,3]. In particular, the work of Moore and Meystre [2] describes the Rayleigh scattering in a

\footnotetext{
${ }^{*}$ Corresponding author. Fax: +39-2-2392-208.

E-mail address: nicola.piovella@mi.infn.it (N. Piovella).
}

BEC using a model which extends the collective atomic recoil laser (CARL) model originally proposed by Bonifacio et al. [4-7] to include a quantum-mechanical description of the centre-of-mass motion of the atoms in the condensate $[8,9]$. The conclusions of Ref. [8] were that the original CARL theory, which treats the atomic centre-of-mass motion classically, fails when the temperature of the atomic sample is below the recoil temperature $T_{\mathrm{r}}=\hbar \omega_{\mathrm{r}} / k_{\mathrm{B}}$, where $\omega_{\mathrm{r}}=\hbar|\vec{q}|^{2} / 2 m$ is the recoil frequency, $m$ is the atomic mass, $\vec{q}=\vec{k}-\vec{k}_{\mathrm{s}}$ is the difference between the pump and scattered wave vectors and $k_{\mathrm{B}}$ is Boltzmann's constant. However, the cubic dispersion relation derived in Ref. [8] reduces to that of the original semiclassical CARL model for large atomic densities. More specifically, 
the quantum corrections to the classical motion are negligibly small when the CARL parameter $\rho$ in the free electron laser (FEL) limit [6,7], roughly interpreted as the average number of photons scattered per atom, is much greater than one. This suggests that a fully quantum-mechanical description of the atomic centre-of-mass motion may not be necessary in order to describe the main experimental results of Ref. [1], i.e. the temporal evolution of the scattered light intensity and the spatial grating in the condensate. We are aware that a semiclassical theory is necessarily limited in its description of the radiation statistics and the quantum degenerate nature of the condensate, which require a full quantum analysis. Nevertheless, we consider the semiclassical approach useful in order to give an intuitive description of the physical mechanism underlying the observed effects. We stress however that in spite of its simplicity, the semiclassical model produces good quantitative agreement with the experimental results of Ref. [1].

\section{Model}

The model described is bidimensional and semiclassical. We represent the cigar-shaped atomic sample as an ellipsoid with length $L$ and diameter $W$, where $L \gg W$ as shown in Fig. 1. The sample is exposed to a classical plane wave radiation electric field $\vec{E}_{0}(y, t)=\hat{x} \mathscr{E}_{0} \mathrm{e}^{\mathrm{i} k(y-c t)}+$ c.c., polarised along the $\hat{x}$-axis and incident along the axis $\hat{y}$, with $\mathscr{E}_{0}$ real and constant and where $k=\omega / c$. We assume that, due to the elongated geometry of the atomic sample, the scattering along the $\hat{z}$-axis dominates over that in other directions, as has been explicitly discussed in Ref. [2], where a full multi-mode theory has shown that mode competition results in light scattering only within the geometric angle $W / L$. Hence, we assume that the scattered radiation consists of two radiation pulses propagating along the $\hat{z}$-axis, with electric fields polarised as the incident field:

$\vec{E}(z, t)=\hat{x}\left[\mathscr{E}_{1}(z, t) \mathrm{e}^{\mathrm{i} k(z-c t)}+\mathscr{E}_{2}(z, t) \mathrm{e}^{-\mathrm{i} k(z+c t)}+\right.$ c.c. $]$,

where $\mathscr{E}_{1,2}(z, t)$ are slowly varying complex amplitudes.

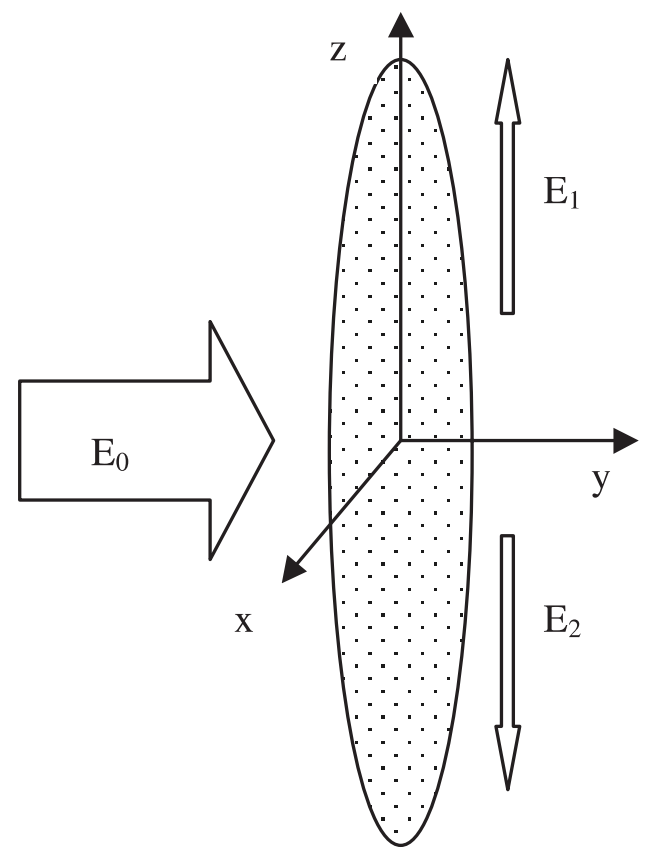

Fig. 1. The geometry of the scattering experiment. The filled ellipsoid, representing the atomic condensate with dimensions $L$ and $W$, is illuminated with a single off-resonant laser beam of electric field $E_{0}$ polarised along the $\hat{x}$-axis and propagating along the $\pm \hat{z}$-axis. The geometry favours the emission of the oppositely directed superradiant pulses $E_{1}$ and $E_{2}$ along the major axis of the condensate.

The atomic sample is described as a collisionless gas of atoms, each with two internal energy levels. The internal evolution of each atom is described by the density matrix elements $\rho_{m n}(m, n=1,2)$ for the lower, (1), and upper, (2), levels. The offdiagonal elements $\rho_{12}=\rho_{21}^{*}$ describe the dipole moment induced by the radiation fields via the relation $\vec{d}=\hat{x} \mu\left(\rho_{12}+\right.$ c.c. $)$, where $\mu$ is the dipole matrix element. The diagonal elements $\rho_{11}$ and $\rho_{22}$ describe the probability of an atom being in the lower or in the upper level, respectively. The offdiagonal elements may be described conveniently as a sum of three polarisation waves:

$$
\begin{aligned}
\rho_{12}= & S_{0}(z, t) \mathrm{e}^{\mathrm{i} k(y-c t)}+S_{1}(z, t) \mathrm{e}^{\mathrm{i} k(z-c t)} \\
& +S_{2}(z, t) \mathrm{e}^{-\mathrm{i} k(z+c t)},
\end{aligned}
$$

where $S_{k}(z, t)(k=0,1,2)$ are slowly varying complex functions. The dipole moment of each atom contributes to the macroscopic polarisation 
of the atomic sample described by $\vec{P}=n(\vec{x}) \vec{d}$, where $n(\vec{x})$ is the atomic density. This polarisation is a source for the radiation field via Maxwell's wave equation which yields, in the usual slowly varying envelope approximation,

$$
\begin{gathered}
\left(\frac{\partial \mathscr{E}_{1}}{\partial t}+c \frac{\partial \mathscr{E}_{1}}{\partial z}\right) \mathrm{e}^{\mathrm{i} k z}+\left(\frac{\partial \mathscr{E}_{2}}{\partial t}-c \frac{\partial \mathscr{E}_{2}}{\partial z}\right) \mathrm{e}^{-\mathrm{i} k z} \\
=\frac{\mathrm{i} \omega \mu}{2 \epsilon_{0}} n(\vec{x})\left\{S_{0} \mathrm{e}^{\mathrm{i} k y}+S_{1} \mathrm{e}^{\mathrm{i} k z}+S_{2} \mathrm{e}^{-\mathrm{i} k z}\right\},
\end{gathered}
$$

where we have neglected the terms proportional to $\mathrm{e}^{ \pm 2 \mathrm{i} \omega t}$. We assume that the atomic sample can be described as a collection of $N$ point particles with positions $\vec{x}_{j}$, so that $n(\vec{x})=\sum_{j=1}^{N} \delta^{(3)}\left(\vec{x}-\vec{x}_{j}\right)$. Multiplying both sides by $\mathrm{e}^{\mathrm{Fi} k z}$ and integrating over the $\hat{z}$-axis from $z-\Delta z / 2$ to $z+\Delta z / 2$, where $\Delta z=\lambda / 2$, Eq. (3) yields

$$
\begin{aligned}
\left(\frac{\partial \mathscr{E}_{1,2}}{\partial t} \pm c \frac{\partial \mathscr{E}_{1,2}}{\partial z}\right) \Delta z= & \frac{\mathrm{i} \omega \mu}{2 \epsilon_{0}} \sum_{j=1}^{N}\left\{S_{0} \mathrm{e}^{\mathrm{i} k\left(y \mp z_{j}\right)}+S_{1,2}\right. \\
& \left.+S_{2,1} \mathrm{e}^{\mp 2 \mathrm{i} k z_{j}}\right\} \delta\left(x-x_{j}\right) \delta\left(y-y_{j}\right),
\end{aligned}
$$

where the upper sign corresponds to the first subscript and we have assumed the field amplitudes $\mathscr{E}_{1,2}$ are spatially slowly varying over $\Delta z$. Assuming also that $\mathscr{E}_{1,2}$ are independent of $x$ and $y$, we can integrate on the plane $(x, y)$ over the section $A=\pi W^{2} / 4$ of the condensate, so that Eq.

(4) becomes

$$
\begin{gathered}
\left(\frac{\partial \mathscr{E}_{1,2}}{\partial t} \pm c \frac{\partial \mathscr{E}_{1,2}}{\partial z}\right)=\frac{\mathrm{i} \omega \mu \bar{n}}{2 \epsilon_{0}}\left\langle S_{0} \mathrm{e}^{\mathrm{i} k(y \mp z)}+S_{1,2}\right. \\
\left.+S_{2,1} \mathrm{e}^{\mp 2 \mathrm{i} k z}\right\rangle,
\end{gathered}
$$

where $\bar{n}=N / A \Delta z$ is the average density and $\langle.\rangle=.(1 / N) \sum_{j=1}^{N}(. .)_{j}$.

In this model the atomic centre-of-mass motion is treated classically, with each atom described as a point particle with a given position and momentum. The radiation fields drive the centre-of-mass motion of the atoms via the force

$\vec{F}=\left(0, \vec{d} \cdot \frac{\partial\left(\vec{E}_{0}+\vec{E}\right)}{\partial y}, \vec{d} \cdot \frac{\partial\left(\vec{E}_{0}+\vec{E}\right)}{\partial z}\right)$.

Neglecting the fast-varying temporal terms, the equations for the atomic velocity components are:

$$
\begin{gathered}
m \frac{\mathrm{d} v_{y}}{\mathrm{~d} t}=\mathrm{i} k \mu \mathscr{E}_{0}\left[S_{0}^{*}+S_{1}^{*} \mathrm{e}^{\mathrm{i} k(y-z)}+S_{2}^{*} \mathrm{e}^{\mathrm{i} k(y+z)}-\text { c.c. }\right] \\
m \frac{\mathrm{d} v_{z}}{\mathrm{~d} t}=\mathrm{i} k \mu\left\{S_{1}^{*} \mathscr{E}_{1}-S_{2}^{*} \mathscr{E}_{2}+S_{2}^{*} \mathscr{E}_{1} \mathrm{e}^{2 \mathrm{i} k z}-S_{1}^{*} \mathscr{E}_{2} \mathrm{e}^{-2 \mathrm{i} k z}\right. \\
\left.+S_{0}^{*}\left[\mathscr{E}_{1} \mathrm{e}^{\mathrm{i} k(z-y)}-\mathscr{E}_{2} \mathrm{e}^{-\mathrm{i} k(y+z)}\right]-\text { c.c. }\right\} .
\end{gathered}
$$

We assume that the detuning $\delta=\omega-\omega_{\mathrm{a}}$ between the optical fields and the atomic resonance is much larger than the natural line width of the atomic transition, $\gamma$, so that the atoms always remain in their lower internal energy states $\left(\rho_{11} \approx 1\right.$ and $\rho_{22} \approx 0$ ). Moreover, assuming that the scattering time scale is much longer than the relaxation time $\gamma^{-1}$, we can adiabatically eliminate the atomic polarisations, i.e. $S_{k}=\mathrm{i}(\mu / \hbar) \mathscr{E}_{k} /(\gamma+\mathrm{i} \delta) \approx \Omega_{k} / 2 \delta$, where $\Omega_{k}=2 \mu \mathscr{E}_{k} / \hbar,\left|\Omega_{k}\right|$ is the Rabi frequency for the field $k$ and $k=0,1,2$. With these approximations, Eqs. (6) and (7) yield:

$$
m \frac{\mathrm{d} v_{y}}{\mathrm{~d} t}=-\mathrm{i} \hbar k\left(\Omega_{0} / 4 \delta\right)\left[\Omega_{1} \mathrm{e}^{\mathrm{i} k(z-y)}-\Omega_{2}^{*} \mathrm{e}^{\mathrm{i} k(z+y)}-\text { c.c. }\right],
$$

$$
\begin{aligned}
m \frac{\mathrm{d} v_{z}}{\mathrm{~d} t}= & \mathrm{i} \hbar k\left(\Omega_{0} / 4 \delta\right)\left[\Omega_{1} \mathrm{e}^{\mathrm{i} k(z-y)}+\Omega_{2}^{*} \mathrm{e}^{\mathrm{i} k(z+y)}-\text { c.c. }\right] \\
& +\mathrm{i}(\hbar k / 2 \delta)\left[\Omega_{1} \Omega_{2}^{*} \mathrm{e}^{2 \mathrm{i} k z}-\text { c.c. }\right] .
\end{aligned}
$$

It is seen that the interference between the pump and the scattered fields forms two bidimensional periodic potentials $V_{1,2}(y, z) \propto\left|\mathscr{E}_{0} \mathscr{E}_{1,2}\right| \cos [k(z \mp$ $\left.y) \pm \phi_{1,2}\right]$ in the plane $(\hat{y}, \hat{z})$, where $\phi_{1,2}$ are the phases of the complex amplitudes $\mathscr{E}_{1,2}$. A weaker $1 \mathrm{D}$ potential $V_{3}(z) \propto\left|\mathscr{E}_{1} \mathscr{E}_{2}\right| \cos \left[2 k z+\phi_{1}-\phi_{2}\right]$ forms along the $\hat{z}$-axis due to the interference of the two counterpropagating scattered fields. If the pump intensity is large enough, we can assume $\mathscr{E}_{0} \gg \mathscr{E}_{1,2}$ and neglect the ponderomotive potential $V_{3}$. Then, Eqs. (8), (9) and (5) can be conveniently written in the following dimensionless form [5]:

$\frac{\mathrm{d} \theta_{1,2}}{\mathrm{~d} \bar{t}}=p_{1,2}$,

$\frac{\mathrm{d} p_{1,2}}{\mathrm{~d} \bar{t}}=\mp\left[A_{1,2} \mathrm{e}^{ \pm \mathrm{i} \theta_{1,2}}+\right.$ c.c. $]$,

$\frac{\partial A_{1,2}}{\partial \bar{t}} \pm \frac{\partial A_{1,2}}{\partial \bar{z}}=\left\langle\mathrm{e}^{\mp \mathrm{i} \theta_{1,2}}\right\rangle$, 
where $\theta_{1,2}=k(z \mp y), p_{1,2}=(m / \hbar k \rho)\left(v_{z} \mp v_{y}\right)$ and $A_{1,2}=-2 \mathrm{i}\left(\epsilon_{0} / \hbar \omega \bar{n} \rho\right)^{1 / 2} \mathscr{E}_{1,2}$ are scaled atomic position, atomic momentum and field amplitude variables respectively. The dimensionless time and space coordinates, $\bar{t}=\omega_{\mathrm{r}} \rho t$ and $\bar{z}=\omega_{\mathrm{r}} \rho z / c$, are scaled in terms of the collective recoil bandwidth, $\rho \omega_{\mathrm{r}}$, where $\omega_{\mathrm{r}}=\hbar k^{2} / m$ is the single-atom recoil frequency and $\rho=\left(\Omega_{0} / 2 \delta\right)^{2 / 3}\left(\omega \mu^{2} \bar{n} / \epsilon_{0} \omega_{\mathrm{r}}^{2} \hbar\right)^{1 / 3}$ is the dimensionless CARL parameter [6,7]. At $\bar{t}=0$, the atoms are assumed to be randomly distributed in position and have zero momentum, and the amplitudes of the scattered fields are set to zero.

\section{Analysis}

In this simple model the two scattered fields are uncoupled and symmetric. For each field $(1,2)$ individually, Eqs. (10)-(12) are formally identical to those which describe pulse propagation in a high gain FEL [7]. It is already known that they admit a self-similar solution of the form $A_{1,2}(\bar{z}, \bar{t})=$ $\pm \bar{z} \mathscr{A}(u)$, where $u=\sqrt{|\bar{z}|}(\bar{t} \mp \bar{z})$ and $\mathscr{A}(u)$ is the solution of a set of ordinary differential equations [10]. This self-similar solution describes the superradiant emission of radiation pulses whose duration decreases in proportion to the fourth root of the peak intensity. The pulse shape can be approximated by a hyperbolic secant function, followed by some non-linear 'ringing', similar to that which occurs in superfluorescence from inverted two-level atoms [11].

A simpler model can be obtained by approximating the spatial derivative in the field equation (12) by a damping term [13] i.e.

$\frac{\mathrm{d} A_{1,2}}{\mathrm{~d} \bar{t}}=\left\langle\mathrm{e}^{\mp \mathrm{i} \theta_{1,2}}\right\rangle-\kappa A_{1,2}$,

where $\kappa=c / 2 \omega_{\mathrm{r}} \rho L$ and $L / c$ is the transit time of the photon along the major axis of the condensate. In this approximation, the finite interaction time due to the escape of radiation from the atomic sample is represented by an incoherent decay of the field amplitude in the sample at a rate $c / 2 L$, half the inverse of the radiation 'lifetime' in the atomic sample. A more general treatment where the radiation is scattered in a direction making an angle $\psi$ with respect to the $\hat{z}$-axis should give $\kappa \approx\left(c / 2 \omega_{\mathrm{r}} \rho\right)[|\sin \psi| / W+|\cos \psi| / L]$ [12]. As
$L \gg W$, the radiation is least strongly damped along the major axis of the sample.

An approximate solution to Eqs. (10), (11) and (13) can be found assuming $\kappa \gg 1$ and adiabatically eliminating the field variables, i.e. $A_{1,2} \approx$ $\kappa^{-1}\left\langle\exp \left[\mp \mathrm{i} \theta_{1,2}\right]\right\rangle$. In this limit, the rate of change of the average scaled momentum is $(\mathrm{d} / \mathrm{d} \bar{t})\left\langle p_{1,2}\right\rangle=$ $\mp 2 \kappa\left|A_{1,2}\right|^{2}$. A third-order analysis of the equations in the mean-field limit (i.e. with radiation propagation modelled by the damping term) gives the following approximate solution:

$\left|A_{1,2}\right|^{2} \approx \frac{1}{2 \kappa^{2}} \operatorname{sech}^{2}\left[\left(\bar{t}-\bar{t}_{\mathrm{D}}\right) / \sqrt{2 \kappa}\right]$

and

$\left\langle p_{1,2}\right\rangle \approx \mp \sqrt{\frac{2}{\kappa}}\left\{1+\tanh \left[\left(\bar{t}-\bar{t}_{\mathrm{D}}\right) / \sqrt{2 \kappa}\right]\right\}$,

where $\bar{t}_{\mathrm{D}}=-\sqrt{2 \kappa} \ln \left(\left|b_{0}\right| / \sqrt{2}\right)$ is the delay time of the peak and $b_{0}=\left\langle\exp \left[\mp \mathrm{i} \theta_{1,2}(\bar{t}=0)\right]\right\rangle$ is the initial bunching, which can be assumed to be $\sim 1 / \sqrt{N}$ for a condensate of $N$ atoms. In the linear regime the exponential gain is

$$
\begin{aligned}
G & =\omega_{\mathrm{r}} \rho \sqrt{2 / \kappa} \\
& =(3 \gamma / \delta) \sqrt{\left(2 I_{0} N / m \omega\right)\left(\lambda^{2} / A\right)},
\end{aligned}
$$

whereas the peak value of the scattered intensity is $I_{\text {peak }}=(\gamma / \delta)^{2}\left[(3 / 2 \pi)\left(\lambda^{2} / A\right) N\right]^{2} I_{0}$,

where $I_{0}=2 c \epsilon_{0}\left|\mathscr{E}_{0}\right|^{2}$ is the pump intensity, $A$ is the cross-sectional area of the condensate and $\gamma=$ $\mu^{2} k^{3} / 6 \pi \hbar \epsilon_{0}$ is the natural decay rate of the atomic transition.

\section{Comparison with the MIT experiment}

In the MIT experiment, a sodium BEC was exposed to a single off-resonant laser pulse red detuned by $\delta / 2 \pi=1.7 \mathrm{GHz}$ from the $3 \mathrm{~S}_{1 / 2} \rightarrow$ $3 \mathrm{P}_{3 / 2}$ transition, with $\lambda=0.589 \mu \mathrm{m}$ and natural width $\gamma=0.31 \times 10^{8} \mathrm{~s}^{-1}$. The recoil frequency is $\omega_{\mathrm{r}}=3 \times 10^{5} \mathrm{~s}^{-1}$. We assume that the condensate had a diameter of $20 \mu \mathrm{m}$ and a length of $200 \mu \mathrm{m}$, approximately $N=5 \times 10^{5}$ atoms participate in the emission of a scattered radiation pulse. The dimensionless parameters are $\rho \approx 44 \times I_{0}^{1 / 3}$ and 
$\kappa \approx 5.5 \times 10^{4} \times I_{0}^{-1 / 3}$, where $I_{0}$ is the pump intensity in $\mathrm{mW} \mathrm{cm}^{-2}$. As $I_{0}>1$ and consequently $\rho \gg 1$, the results of Ref. [8] indicate that quantum effects due to atomic diffraction should be negligibly small for this experiment, even though $T \ll T_{\mathrm{r}}$. As $\kappa \gg 1$, the atoms emit two superradiant pulses along the major axis of the condensate. The gain is approximately $G \approx 82 \times \sqrt{I_{0}}$, where $G$ is given in $\mathrm{ms}^{-1}$, whereas the peak occurs after a time $t_{\mathrm{D}}=\ln (2 N) / G \approx\left(170 / \sqrt{I_{0}}\right) \quad \mu \mathrm{s}$, in good agreement with the measured values of Ref. [1]. Furthermore, from Eq. (15) the modulus of the average atomic velocity is $v=(\hbar k / m) \rho\left|\left\langle p_{1,2}\right\rangle\right| \approx$ $(\lambda / 2 \pi) G \approx 0.7 \sqrt{I_{0}} \mathrm{~cm} \mathrm{~s}^{-1}$. Fig. 2 shows the temporal evolution of the main peak of the scattered intensity, as given by the approximate formula (14), for the parameters of the MIT experiment and three different values of the incident intensity, $3.8 \mathrm{~mW} \mathrm{~cm}^{-2}$ (solid line), $2.4 \mathrm{~mW} \mathrm{~cm}^{-2}$ (dashed line) and $1.4 \mathrm{~mW} \mathrm{~cm}^{-2}$ (dotted line).

In addition to the temporal evolution of the scattered radiation pulses, there are other predictions of this semiclassical model which are consistent with the results of the MIT experiment: Firstly, superradiance is observable only if the Doppler broadening of the atomic resonance is sufficiently small that $\sigma t_{\text {sr }} \ll 1$, where $\sigma$ is the rms spread of the gaussian spectral distribution and $t_{\mathrm{sr}} \sim 1 / G$ is the superradiant time [11]. The observed spectral width of the Bragg resonance of the BEC of approximately $5 \mathrm{kHz}$ [14] (corresponding to a velocity spread of few $\mathrm{mm} \mathrm{s}^{-1}$ ) yields $\sigma t_{\mathrm{sr}} \sim 0.16 \times I_{0}^{-1 / 2}$. We observe that, using $\sigma=$ $k\left(k_{\mathrm{B}} T / m\right)^{1 / 2}$, a temperature of only $1 \mu \mathrm{K}$ (approximately the BEC transition temperature for the MIT experiment) would increase the frequency spread by a factor of 15 , enough to destroy the superradiant emission. This explains why superradiant emission was observed only at the extremely low temperatures below the threshold for Bose-Einstein condensation [1]. Secondly, superradiant emission parallel and antiparallel to the $\hat{z}$-axis induces an average atomic velocity $\vec{v}_{1,2} \approx$ $(G / k)[\hat{y} \mp \hat{z}]$, respectively at $45^{\circ}$ with respect to the negative (positive) direction of the $\hat{z}$-axis, as observed in the MIT experiment. We assume the existence of two distinct families of atoms interacting with the two independent superradiant

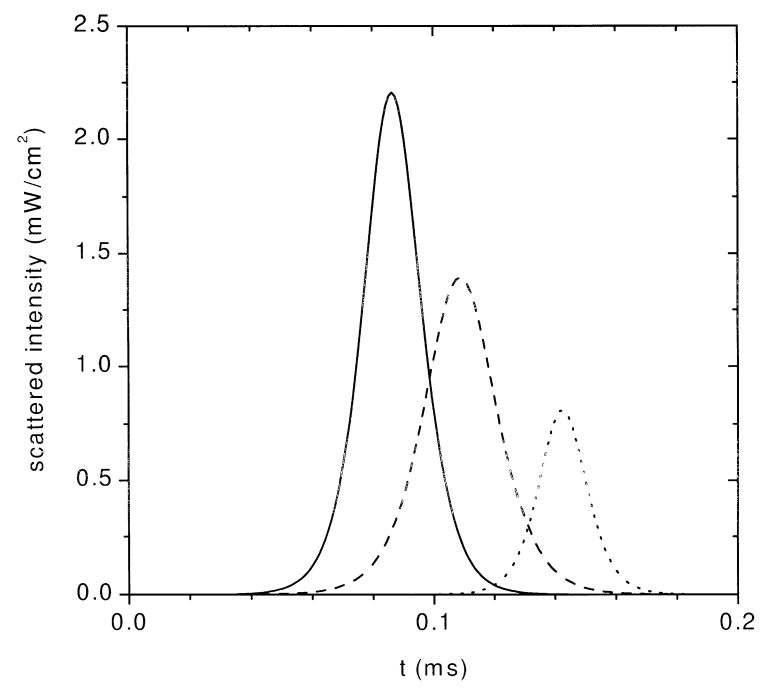

Fig. 2. Temporal evolution of the main peak of the scattered intensity as given by the approximate formula (14), for the parameters of the MIT experiment and three different values of the incident intensity, $3.8 \mathrm{~mW} \mathrm{~cm}^{-2}(-), 2.4 \mathrm{~mW} \mathrm{~cm}^{-2}(---)$ and $1.4 \mathrm{~mW} \mathrm{~cm}^{-2}(\cdots)$.

pulses $A_{1}$ and $A_{2}$. However different orders of atomic velocity, i.e. $\vec{v}_{m, n}=m \vec{v}_{1}+n \vec{v}_{2}$, with $m, n$ integers, have also been observed. More precisely, the orders $(2,0),(-1,1),(0,2),(2,1)$ and $(1,2)$ other than the usual $(1,0)$ and $(0,1)$, have been clearly observed in the experiment after increasing the exposure time to the laser source and letting the atomic cloud expand ballistically after the interaction [1]. The formation of this momentum grating can be explained as a sequential superradiant scattering process in which the atoms emit $m$ pulses along the positive $\hat{z}$ and $n$ pulses along the negative $\hat{z}$-axis, acquiring a total recoil velocity $\vec{v}_{m, n}$. The extremely narrow resonance line allows the atoms to emit up to three sequential superradiantly scattered pulses before $\sigma t \sim 1$, which is consistent with the observation of the atomic momentum distribution.

\section{Conclusions}

In conclusion, we have presented a semiclassical model describing the superradiant Rayleigh scattering from a BEC observed in Ref. [1]. The model is much simpler than those previously used 
to explain the results of Ref. [1] as the atomic centre-of-mass motion is treated classically. The evolution of the scattered intensity and the atomic motion due to recoil as calculated from this simple model are in quantitative agreement with the experimental results. The fact that quantum centre-of-mass effects such as atomic diffraction are negligible is a consequence of the high density of the condensate. In our model the BEC is essentially described as a collisionless Doppler-free atomic gas. The results presented here suggest that together with its high density, the most important property of the condensate with regard to superradiant light scattering is its very low temperature rather than its quantum degenerate nature. In this respect the situation is similar to that of ultraslow propagation of light in a BEC [15]. Subsequent observations of ultraslow propagation in a hot vapour [16] demonstrated that the significant property of the BEC was that it was a Dopplerfree optical medium rather than a quantum degenerate one.

\section{Acknowledgements}

The authors would like to thank the Royal Society of Edinburgh for support of G.R.M.R. and the EPSRC for support of B.M $\mathrm{M}^{\mathrm{c}} \mathrm{N}$.

\section{References}

[1] S. Inouye, A.P. Chikkatur, D.M. Stamper-Kurn, J. Stenger, D.E. Pritchard, W. Ketterle, Science 285 (1999) 571.

[2] M.G. Moore, P. Meystre, Phys. Rev. Lett. 83 (1999) 5202.

[3] O.E .Mustecaplioglu, L.You, http://xxx.lanl.gov/abs/condmatt/0002390.

[4] R. Bonifacio, L. De Salvo Souza, Nucl. Instrum. Meth. Phys. Rev. A 341 (1994) 360.

[5] R. Bonifacio, L. De Salvo Souza, L.M. Narducci, E.J. D’Angelo, Phys. Rev. A 50 (1994) 1716.

[6] R. Bonifacio, L. De Salvo, Appl. Phys. B 60 (1995) S233.

[7] R. Bonifacio, G.RM. Robb, B.W.J. McNeil, Phys. Rev. A 56 (1997) 912.

[8] M.G. Moore, P. Meystre, Phys. Rev. A 58 (1998) 3248.

[9] M.G. Moore, O. Zobay, P. Meystre, Phys. Rev. A 60 (1999) 1491.

[10] R. Bonifacio, L. De Salvo Souza, P. Pierini, N. Piovella, Nucl. Instrum. Meth. Phys. Res. A 296 (1990) 358.

[11] R. Bonifacio (Ed.), Dissipative Systems in Quantum Optics, Springer, Berlin, 1982.

[12] R. Bonifacio, L.A. Lugiato, Phys. Rev. A 11 (1975) 1507.

[13] R. Bonifacio, F. Casagrande, J. Opt. Soc. Am. B 2 (1985) 250.

[14] J. Stenger, S. Inouye, A.P. Chikkatur, D.M. StamperKurn, D.E. Pritchard, W. Ketterle, Phys. Rev. Lett. 82 (1999) 4569.

[15] L.V. Hau, S.E. Harris, Z. Dutton, C.H. Behroozi, Nature 397 (1999) 594.

[16] M.M. Kash, V.A. Sautenkov, A.S. Zibrov, L. Hollberg, G.R. Welch, M.D. Lukin, Y. Rostovsev, E.S. Fry, M.O. Scully, Phys. Rev. Lett. 82 (1999) 5229. 